\title{
Front Matter: Volume 7214
}

, "Front Matter: Volume 7214," Proc. SPIE 7214, Ultrafast Phenomena in Semiconductors and Nanostructure Materials XIII, 721401 (11 March 2009); doi: $10.1117 / 12.824586$

Event: SPIE OPTO: Integrated Optoelectronic Devices, 2009, San Jose, SPIE. California, United States 


\title{
PROCEEDINGS OF SPIE
}

\section{Ultrafast Phenomena in Semiconductors and Nanostructure Materials XIII}

\author{
Kong-Thon Tsen \\ Jin-Joo Song \\ Markus Betz \\ Abdulhakem Y. Elezzabi \\ Editors \\ 25-28 January 2009 \\ San Jose, California, United States \\ Sponsored by \\ SPIE \\ Cosponsored by \\ PolarOnyx, Inc. (United States) \\ Published by \\ SPIE
}


The papers included in this volume were part of the technical conference cited on the cover and title page. Papers were selected and subject to review by the editors and conference program committee. Some conference presentations may not be available for publication. The papers published in these proceedings reflect the work and thoughts of the authors and are published herein as submitted. The publisher is not responsible for the validity of the information or for any outcomes resulting from reliance thereon.

Please use the following format to cite material from this book:

Author(s), "Title of Paper," in Ultrafast Phenomena in Semiconductors and Nanostructure Materials XIII, edited by Kong-Thon Tsen, Jin-Joo Song, Markus Betz, Abdulhakem Y. Elezzabi, Proceedings of SPIE Vol. 7214 (SPIE, Bellingham, WA, 2009) Article CID Number.

ISSN 0277-786X

ISBN 9780819474605

Published by

SPIE

P.O. Box 10, Bellingham, Washington 98227-0010 USA

Telephone +1 3606763290 (Pacific Time) · Fax +1 3606471445

SPIE.org

Copyright (c) 2009, Society of Photo-Optical Instrumentation Engineers

Copying of material in this book for internal or personal use, or for the internal or personal use of specific clients, beyond the fair use provisions granted by the U.S. Copyright Law is authorized by SPIE subject to payment of copying fees. The Transactional Reporting Service base fee for this volume is $\$ 18.00$ per article (or portion thereof), which should be paid directly to the Copyright Clearance Center (CCC), 222 Rosewood Drive, Danvers, MA 01923. Payment may also be made electronically through CCC Online at copyright.com. Other copying for republication, resale, advertising or promotion, or any form of systematic or multiple reproduction of any material in this book is prohibited except with permission in writing from the publisher. The CCC fee code is 0277-786X/09/\$18.00.

Printed in the United States of America.

Publication of record for individual papers is online in the SPIE Digital Library.

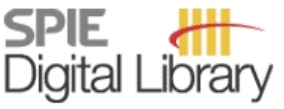

SPIEDigitalLibrary.org

Paper Numbering: Proceedings of SPIE follow an e-First publication model, with papers published first online and then in print and on CD-ROM. Papers are published as they are submitted and meet publication criteria. A unique, consistent, permanent citation identifier (CID) number is assigned to each article at the time of the first publication. Utilization of CIDs allows articles to be fully citable as soon they are published online, and connects the same identifier to all online, print, and electronic versions of the publication. SPIE uses a six-digit CID article numbering system in which:

- The first four digits correspond to the SPIE volume number.

- The last two digits indicate publication order within the volume using a Base 36 numbering system employing both numerals and letters. These two-number sets start with 00, 01, 02, 03, 04 , 05, 06, 07, 08, 09, OA, OB ... 0Z, followed by 10-1Z, 20-2Z, etc.

The CID number appears on each page of the manuscript. The complete citation is used on the first page, and an abbreviated version on subsequent pages. Numbers in the index correspond to the last two digits of the six-digit CID number. 


\section{Contents}

ix Conference Committee

\section{CARRIER AND EXCITON DYNAMICS I}

721403 High-resolution temporal and spatial photoluminescence measurement of a multiple-quantum-well structure at room temperature (Invited Paper) [7214-02] H. An, J. Kim, D. Kim, E. Lee, Yonsei Univ. (Korea, Republic of); D.-S. Shin, Hanyang Univ. (Korea, Republic of); H. Doh, S.-H. Park, Yonsei Univ. (Korea, Republic of)

721404 Generation of injection currents in (110)-oriented GaAs quantum wells: experimental observation and development of a microscopic theory (Invited Paper) [7214-03] M. Bieler, K. Pierz, U. Siegner, Physikalisch-Technische Bundesanstalt (Germany); P. Dawson, The Univ. of Manchester (United Kingdom); H. T. Duc, J. Förstner, T. Meier, Univ. Paderborn (Germany)

721405 Carrier dynamics and photoexcited emission efficiency of ZnO:Zn phosphor powders (Invited Paper) [7214-04]

J. V. Foreman, H. O. Everitt, Duke Univ. (United States) and U.S. Army Aviation and Missile Research, Development, and Engineering Ctr. (United States); J. Yang, J. Liu, Duke Univ. (United States)

\section{PHONONS}

721406 Imaging carrier and phonon transport in Si using ultrashort optical pulses (Invited Paper) [7214-05]

D. H. Hurley, Idaho National Lab. (United States); O. B. Wright, O. Matsuda, Hokkaido Univ. (Japan); B. E. McCandless, Univ. of Delaware (United States); S. Shinde, Sandia National Labs. (United States)

721407 Ultrafast high strain rate acoustic wave measurements at high static pressure in a diamond anvil cell (Invited Paper) [7214-06]

M. R. Armstrong, J. C. Crowhurst, E. J. Reed, J. M. Zaug, Lawrence Livermore National Lab. (United States)

721408 Picosecond shear waves in nano-sized solids and liquids (Invited Paper) [7214-07] T. Pezeril, LPEC, CNRS, Univ. du Maine (France) and Massachusetts Institute of Technology (United States); C. Klieber, Massachusetts Institute of Technology (United States); S. Andrieu, LPM, CNRS, Univ. H. Poincaré (France); D. Chateigner, Crismat-Ensicaen, CNRS, Univ. de Caen Basse-Normandie (France); K. A. Nelson, Massachusetts Institute of Technology (United States) 
7214 OA Ultrafast far-infrared optics of carbon nanotubes (Invited Paper) [7214-09]

C. Frischkorn, T. Kampfrath, L. Perfetti, K. von Volkmann, M. Wolf, Freie Univ. Berlin (Germany)

\section{NANOSTRUCTURES AND NANOPHOTONICS II}

7214 OF Photoinduced ultrafast structural dynamics of nanomaterials (Invited Paper) [7214-14] J. Tang, P. Yu, P.-T. Tai, Research Ctr. For Applied Sciences (Taiwan); S.-H. Lin, Institute of Atomic and Molecular Sciences (Taiwan)

$7214 \mathrm{OH} \quad$ Electromagnetic wave funneling through nano-gaps and nano-antennas (Invited Paper) [7214-16]

D. S. Kim, M. A. Seo, H. R. Park, J. S. Kyoung, J. W. Lee, Seoul National Univ. (Korea, Republic of); O. K. Suwal, S. S. Choi, Sun Moon Univ. (Korea, Republic of)

\section{APPLICATIONS I}

$72140 \mathrm{~J} \quad$ Laser ablation of organic materials for discrimination of bacteria in an inorganic background (Invited Paper) [7214-18]

M. Baudelet, M. Boueri, J. Yu, Univ. Lyon I, LASIM, CNRS (France); X. Mao, S. S. Mao, R. Russo, Lawrence Berkeley National Lab. (United States)

7214 OK Control of ultrafast pulse propagation in semiconductor components (Invited Paper) [7214-19]

M. van der Poel, P. L. Hansen, J. Mørk, Technical Univ. of Denmark (Denmark)

\section{THz SPECTROSCOPY AND APPLICATIONS I}

$72140 \mathrm{~N}$ Terahertz conductivity of magnetoexcitons and electrons in semiconductor nanostructures (Invited Paper) [7214-22]

J. Lloyd-Hughes, J. Faist, ETH Zürich (Switzerland); H. E. Beere, D. A. Ritchie, Univ. of Cambridge (United Kingdom); L. Sirbu, I. M. Tiginyanu, Technical Univ. of Moldova (Moldova) and Institute of Applied Physics (Moldova); S. K. M. Merchant, M. B. Johnston, Univ. of Oxford (United Kingdom)

721400 Chirality and optical activity: a terahertz time-domain spectroscopy investigation (Invited Paper) [7214-23]

A. Y. Elezzabi, S. Sederberg, Univ. of Alberta (Canada)

7214 OP A new mechanism for observation of THz acoustic waves: coherent THz radiation emission (Invited Paper) [7214-24]

E. J. Reed, M. R. Armstrong, Lawrence Livermore National Lab. (United States); K.-Y. Kim, J. H. Glownia, Los Alamos National Lab. (United States); W. M. Howard, Lawrence Livermore National Lab. (United States); E. L. Piner, J. C. Roberts, Nitronex Corp. (United States)

$72140 Q \quad$ Photo-induced insulator-metal phase transition observed by the terahertz pump-probe spectroscopy (Invited Paper) [7214-25]

M. Nakajima, N. Takubo, Z. Hiroi, Y. Ueda, T. Suemoto, The Univ. of Tokyo (Japan) 
7214 OR Latest developments of ultrafast fiber laser and its material applications (Invited Paper) [7214-26]

G. C. Cho, B. Liu, L. Shah, Z. Liu, Y. Che, J. Xu, IMRA America, Inc. (United States)

7214 OS Monitoring of microplasma formation and filamentation of tightly focused femtosecond laser pulses in dielectrics (Invited Paper) [7214-27]

E. Gaižauskas, Vilnius Univ. (Lithuania); V. Mizeikis, Hokkaido Univ. (Japan) and Shizuoka Univ. (Japan); H. Misawa, Hokkiado Univ. (Japan); S. I. Kudryashov, V. D. Zvorykin, A. A. Ionin, P.N. Lebedev Physical Institute (Russian Federation); S. Juodkazis, Hokkaido Univ. (Japan)

$72140 \mathrm{OU} \quad$ Sub-GHz operation of single-photon emitting diode at $\mathbf{1 . 5 5} \boldsymbol{\mu m}$ (Invited Paper) [7214-29] T. Miyazawa, T. Nakaoka, The Univ. of Tokyo (Japan); S. Okumura, S. Hirose, K. Takemoto, M. Takatsu, Fujitsu Labs., Ltd. (Japan); T. Usuki, The Univ. of Tokyo (Japan); N. Yokoyama, Fujitsu Labs., Ltd. (Japan); Y. Arakawa, The Univ. of Tokyo (Japan)

7214 OV Pulse and amplifier dynamics in high energy fiber optic ultrashort pulse laser systems (Invited Paper) [7214-30]

M. Mielke, D. Gaudiosi, K. Kim, T. Yilmaz, M. Greenberg, S. Tong, X. Gu, M. Geusen, R. Cline, M. Slovick, N. Allen, M. Manning, B. Schuler, S. Sapers, Raydiance, Inc. (United States)

\section{THz SPECTROSCOPY AND APPLICATIONS II}

7214 OW Nonlinear THz response of $\boldsymbol{n}$-type GaAs (Invited Paper) [7214-31]

M. Woerner, W. Kuehn, P. Gaal, K. Reimann, T. Elsaesser, Max-Born-Institut für Nichtlineare Optik und Kurzzeitspektroskopie (Germany); R. Hey, Paul-Drude-Institut für Festkörperelektronik (Germany)

$72140 Y \quad$ THz emission from coherent plasmons in InAs nanowires (Invited Paper) [7214-33]

D. V. Seletskiy, M. P. Hasselbeck, M. Sheik-Bahae, The Univ. of New Mexico (United States): J. G. Cederberg, A. A. Talin, Sandia National Labs. (United States)

$72140 Z$ Terahertz spectroscopy of two-dimensional subwavelength plasmonic structures (Invited Paper) [7214-34]

A. K. Azad, H.-T. Chen, A. J. Taylor, J. F. O'Hara, Los Alamos National Lab. (United States); J. Han, X. LU, W. Zhang, Oklahoma State Univ. (United States)

\section{KEYNOTE PRESENTATION AND SPINTRONICS}

721412 Memory effects in femtosecond collective spin rotation in ferromagnetic semiconductors (Invited Paper) [7214-37]

J. Wang, lowa State Univ. (United States); I. Cotoros, D. S. Chemla, Lawrence Berkeley National Lab., Univ. of California, Berkeley (United States); X. Liu, J. K. Furdyna, Univ. of Notre Dame (United States); J. Chovan, I. E. Perakis, Univ. of Crete (Greece) 


\section{CARRIER AND EXCITON DYNAMICS II}

721413 Ultrafast carrier dynamics and laser action in ZnO nanowires (Invited Paper) [7214-38] M. A. M. Versteegh, R. E. C. van der Wel, B. J. M. Brenny, B. Zegers, W. Ensing, J. I. Dijkhuis, Univ. Utrecht (Netherlands)

721414 Picosecond scale dynamics of excitons in CdTe-based quantum wells and quantum dots (Invited Paper) [7214-39]

A. Golnik, A. Trajnerowicz, T. Kazimierczuk, P. Kossacki, Univ. of Warsaw (Poland)

721416 Ultrafast electronic transport in low dimensional semiconductor nanostructures (Invited Paper) [7214-41]

H. Choi, Lawrence Berkeley National Lab., Univ. of California, Berkeley (United States) and Univ. of Michigan, Ann Arbor (United States); T. B. Norris, Univ. of Michigan, Ann Arbor (United States); J. Faist, ETH Zürich (Switzerland); F. Capasso, Harvard Univ. (United States)

\section{THZ SPECTROSCOPY AND APPLICATIONS III}

721417 Terahertz metamaterials (Invited Paper) [7214-42]

H.-T. Chen, J. F. O'Hara, A. K. Azad, Los Alamos National Lab. (United States); W. J. Padilla, Boston College (United States); R. D. Averitt, Boston Univ. (United States); A. J. Taylor, Los Alamos National Lab. (United States)

\section{CARRIER AND EXCITON DYNAMICS III}

$72141 \mathrm{~A}$ Hole confinement in quantum islands in $\mathrm{Ga}(\mathrm{AsSb}) / \mathrm{GaAs} /(\mathrm{AIGa}) \mathrm{As}$ heterostructures (Invited Paper) [7214-46]

S. Horst, S. Chatterjee, K. Hantke, P. J. Klar, C. Lange, I. Nemeth, M. Schwalm, W. Stolz, K. Volz, C. Bückers, A. D. Thränhardt, S. W. Koch, W. Rühle, Philipps-Univ. Marburg (Germany);

S. R. Johnson, J.-B. Wang, Y.-H. Zhang, Arizona State Univ. (United States)

\section{CARRIER AND EXCITON DYNAMICS IV}

$72141 G \quad$ Femtosecond carrier dynamics in quasi-one-dimensional topological compounds (Invited Paper) [7214-52]

Y. Toda, K. Shimatake, S. Tanda, Hokkaido Univ. (Japan); J. Onoe, Tokyo Institute of Technology (Japan)

\section{CARRIER AND EXCITON DYNAMICS VI}

$72141 \mathrm{M} \quad$ Ultrafast carrier dynamics in Si and on Si surfaces studied by femtosecond time-resolved two-photon photoemission spectroscopy (Invited Paper) [7214-59]

K. Tanimura, T. Ichibayashi, Osaka Univ. (Japan) 
$72141 \mathrm{~N}$ Two color pump-probe studies of intraminiband relaxation in doped GaAs/AIGaAs superlattices (Invited Paper) [7214-60]

D. Stehr, Univ. of California, Santa Barbara (United States) and Forschungszentrum Dresden-Rossendorf e. V. (Germany); M. Wagner, H. Schneider, M. Helm, Forschungszentrum Dresden-Rossendorf e. V. (Germany); A. M. Andrews, T. Roch, G. Strasser, Technische Univ. Wien (Austria)

\section{POSTER SESSION}

721410 Analysis of confinement loss and single mode property in five layer hexagonal (circular/elliptical) layouts of microstructured photonic crystal fiber [7214-62]

R. Sharma, V. Janyani, Malaviya National Institute of Technology (India)

Author Index 
Downloaded From: https://www.spiedigitallibrary.org/conference-proceedings-of-spie on 26 Apr 2023

Terms of Use: https://www.spiedigitallibrary.org/terms-of-use 


\title{
Conference Committee
}

\author{
Symposium Chair \\ James G. Grote, Air Force Research Laboratory (United States) \\ Symposium Cochair
}

E. Fred Schubert, Rensselaer Polytechnic Institute (United States)

Program Track Chair

James G. Grote, Air Force Research Laboratory (United States)

Conference Chairs

Kong-Thon Tsen, Arizona State University (United States)

Jin-Joo Song, University of California, San Diego (United States)

Conference Cochairs

Markus Betz, Technische Universität München (Germany)

Abdulhakem Y. Elezzabi, University of Alberta (Canada)

Program Committee

Serge Bidnyk, Enablence Inc. (Canada)

Mischa Bonn, FOM Institute for Atomic and Molecular Physics (Netherlands)

Majed Chergui, École Polytechnique Fédérale de Lausanne (Switzerland)

Yujie J. Ding, Lehigh University (United States)

Jan A. Gaj, Uniwersytet Warszawski (Poland)

Michael D. Gerhold, U.S. Army Research Office (United States)

Costas P. Grigoropoulos, University of California, Berkeley (United States)

Rupert Huber, Universität Konstanz (Germany)

Robert A. Kaindl, Lawrence Berkeley National Laboratory (United States)

Christoph Lienau, Carl von Ossietzky Universität Oldenburg (Germany)

Torsten Meier, Universität Paderborn (Germany)

Chi-Kuang Sun, National Taiwan University (Taiwan)

Fabrice Vallee, Université Claude Bernard Lyon 1 (France)

Chih-Chung Yang, National Taiwan University (Taiwan) 
Session Chairs

1 Carrier and Exciton Dynamics I

Kong-Thon Tsen, Arizona State University (United States)

2 Phonons

Kong-Thon Tsen, Arizona State University (United States)

3 Nanostructures and Nanophotonics I

Jin-Joo Song, University of California, San Diego (United States)

$4 \quad$ Nanostructures and Nanophotonics II

Markus Betz, Technische Universität München (Germany)

5 Applications I

Abdulhakem Y. Elezzabi, University of Alberta (Canada)

$6 \mathrm{THz}$ Spectroscopy and Applications I

Markus Betz, Technische Universität München (Germany)

7 Applications II

Kong-Thon Tsen, Arizona State University (United States)

$8 \mathrm{THz}$ Spectroscopy and Applications II

Abdulhakem Y. Elezzabi, University of Alberta (Canada)

9 Keynote Presentation and Spintronics

Jin-Joo Song, University of California, San Diego (United States)

10 Carrier and Exciton Dynamics II

Chih-Chung Yang, National Taiwan University (Taiwan)

11 THz Spectroscopy and Applications III

Abdulhakem Y. Elezzabi, University of Alberta (Canada)

12 Carrier and Exciton Dynamics III

Markus Betz, Technische Universität München (Germany)

13 Carrier and Exciton Dynamics IV

Yujie J. Ding, Lehigh University (United States)

14 Carrier and Exciton Dynamics $\vee$

Markus Betz, Technische Universität München (Germany)

15 Carrier and Exciton Dynamics VI

Markus Betz, Technische Universität München (Germany) 